\title{
COMBINED EFFECTS OF NQ01 PR0187SER OR SULT1A1 ARG213HIS POLYMORPHISM AND SMOKING ON BLADDER CANCER RISK: TWO META-ANALYSES
}

\author{
XIAO-CHUN WANG ${ }^{1}$, JIAN WANG ${ }^{1}$, HUI-HUI TAO ${ }^{2}$, CHAO ZHANG ${ }^{2}$, and LI-FA XU ${ }^{1}$ \\ Medical College of Anhui University of Science and Technology, Huainan, China \\ ${ }^{1}$ Department of Pathogen Biology \\ ${ }^{2}$ Department of Biochemistry
}

\begin{abstract}
Objectives: Cigarette smoking is the major risk factor of bladder cancer via exposure to chemical carcinogens. Nicotinamide adenine dinucleotide phosphate $\left(\mathrm{NADP}^{+}\right)$: quinine oxidoreductase 1 (NQO1) and sulfotransferase 1A1 (SULT1A1) have been reported to involve in the metabolism of polycyclic aromatic hydrocarbons (PAHs) and aromatic amines. Therefore, the risk of bladder cancer (BC) may be influenced by polymorphisms in the genes that modulate metabolic detoxification in particular by interacting with cigarette smoking. Considering the limited power by the individual studies with a relatively small sample size, especially when analyzing the combined effect of polymorphisms in NQO1 and SULT1A1 genes and smoking, these 2 meta-analyses have aimed to clarify the combined effects of them on BC risk by integrating related studies. Material and Methods: Two meta-analyses included 1341 cases and 1346 controls concerning NQO1 Pro187Ser and smoking, and 1921 cases and 1882 controls on SULT1A1 Arg213His and smoking were performed. Odds ratios (OR) and 95\% confidence intervals (CI) were used for assessing the strength of the association. Results: The result has demonstrated that smokers with NQO1 Pro/Ser or Ser/Ser genotypes have a prominent association with the risk of BC as compared with non-smokers with NQO1 Pro/Pro genotype, with OR equal to 3.71 (95\% CI: 2.87-4.78, pheterogeneity $=0.376$ ). Besides, smokers carrying SULT1A1 Arg/Arg genotypes were observed to confer 2.38 fold increased risk of BC (95\% CI: 1.44-3.93, $\mathrm{p}_{\text {heterogeneity }}=0.001$ ) when compared with non-smokers with SULT1A1 Arg/Arg or His/His genotypes. Conclusions: These findings have suggested that the NQO1 Pro187Ser or SULT1A1 Arg213His polymorphism combination with smoking significantly confer susceptibility to BC. Int J Occup Med Environ Health 2017;30(5):791-802
\end{abstract}

Key words:

Polymorphisms, Meta-analysis, NQO1, Urinary bladder neoplasms, Smoking, SULT1A1

\section{INTRODUCTION}

Bladder cancer (BC) has remained the eleventh most common cancer worldwide, accounting for a global incidence rate of 429793 and mortality rate of 165084 in 2012 [1]. The incidence rate of colorectal cancer has still been increasing, especially in the western world. The etiology of bladder cancer has been complicated, with both host genetic variants and environmental factors contributing to its development. Extensive evidence has indicated that several environmental factors, including cigarette smoking, aromatic amines, aniline dyes, nitrates, acrolein, coal, and arnicare are involved in the development of BC [2,3].

Received: February 16, 2016. Accepted: September 22, 2016.

Corresponding author: L.-F. Xu, Medical College of Anhui University of Science and Technology, Department of Pathogen Biology, Taifeng avenue 168, Huainan 232001, China (e-mail: xulifa1999@163.com). 
Among the environmental factors, cigarette smoking has been established as the major risk factor for $\mathrm{BC}$, which confers around two- to four-fold increased risk of BC, according to previous epidemiology studies and the metaanalysis $[4,5]$. It has been estimated that with the elimination of smoking, the incidence of bladder cancer could be reduced by approximately $50 \%$ for men and $25 \%$ for women [6].

The important chemical carcinogens of cigarette smoke, including polycyclic aromatic hydrocarbons, heterocyclic aromatic amines and N-nitroso may induce the process of carcinogenic by several of molecular mechanisms in the development of BC. Most chemical carcinogens contained in cigarettes are metabolized and detoxified by phase II enzymes such as nicotinamide adenine dinucleotide phosphate $\left(\mathrm{NADP}^{+}\right)$: quinine oxidoreductase 1 (NQO1) and sulfotransferase 1A1 (SULT1A1) and are then excreted in urine [7]. The $\mathrm{AD}(\mathrm{P}) \mathrm{H}$ :quinone oxidoreductase (NQO1) is a detoxification enzyme that plays a crucial role in the protection against oxidative damage by preventing the generation of reactive oxygen species and reducing certain chemical carcinogens [8,9].

Besides, SULT1A1 plays an important role in the bioactivation and detoxification of many environmental mutagens and procarcinogens, including chemical carcinogens in cigarettes [10]. Functional polymorphisms of the NQO1 or SULT1A1 gene which influence the activities of the corresponding enzymes might be associated with susceptibility to BC. A common single nucleotide polymorphism in codon 187 of NQO1 (rs1800566, a C to T transition) causes the Pro to Ser amino acid substitution, and the Ser187 variant shows lower enzyme activity as compared with the Pro187 variant $[9,11]$. In addition, a nonsynonymous SNP of SULT1A1 gene has been identified in the codon 213 in exon 7 (rs9282861, a G to A transition), which results in an Arg to His amino acid substitution, and the His213 allele has been shown to have lower enzyme activity and thermal stability [12,13].
Furthermore, great numbers of association studies have been conducted to explore the relationship between NQO1 Pro187Ser or SULT1A1 Arg213His polymorphism and the risk of BC. Moreover, the increased effects of NQO1 Pro187Ser or SULT1A1 Arg213His polymorphism on the risk of various cancers including $\mathrm{BC}$ have been clarified by the previous meta-analyses [14-20]. Importantly, a certain amount of the original study has not only investigated the individual effect of NQO1 Pro187Ser or SULT1A1 Arg213His on the risk of BC but has also examined the genetic effect of NQO1 Pro187Ser or SULT1A1 Arg213His modified by smoking on BC risk. Furthermore, the controversial results have been yielded by the previous studies considering the limited power by the studies with a relatively small sample size especially when analyzing the combined effect of NQO1 Pro187Ser or SULT1A1 Arg213His and smoking. Consequently, it has been necessary to calculate the pooled effect of Pro187Ser or SULT1A1 Arg213His and smoking on the risk of $\mathrm{BC}$ by integrating individual studies. Therefore, we have carried out 2 meta-analyses to derive more precise risk estimations for the combined effects of the 2 polymorphisms on bladder cancer risk.

\section{MATERIAL AND METHODS}

\section{Search strategy and study selection}

We systematically browse through the online electronic databases (PubMed, EMBASE, ISI Web of Science and Chinese Biomedical (CBM) database) for published papers up to June, 2016, using the search terms "bladder cancer," "NQO1," "polymorphism" and "smoking" for the analysis between smoking-NQO1 Pro187Ser interaction and bladder cancer risk. Meanwhile, we have used the following search terms "bladder cancer," "sulfotransferase 1A1," "polymorphism," and "smoking" for the analysis between smoking-SULT1A1 Arg213His interaction and bladder cancer risk. The entire search has been limited to the English and Chinese language papers. 
Additional studies have been supplied by a hand search of the references of retrieved articles and reviews.

The inclusion criteria have been as follows:

- case-control study design,

- genotype frequencies on NQO1 Pro187Ser polymorphisms or SULT1A1 Arg213His stratified by smoking which are permitted to calculate odds ratio (OR) with $95 \%$ confidence interval (CI) for estimation of the combined effects of the 2 polymorphism and smoking,

- the distribution of genotypes in the controls conformed to Hardy-Weinberg equilibrium ( $\mathrm{p}>0.05)$.

No control population, duplicate of previous publication, animal studies, reviews and unpublished reports have been excluded.

\section{Data extraction}

All the included data was extracted independently by 2 reviewers. The following information was extracted from the eligible studies: first author's surname, year of publication, country, ethnicity, source of controls, sample size, genotyping method, smoking status and genotype frequencies in both case and control groups stratified by smoking.

\section{Statistical analysis}

The crude ORs and their $95 \%$ CIs were calculated to assess the strength of the association for the NQO1 Pro187Ser or SULT1A1 Arg213His interaction with smoking in the risk of bladder cancer risk. The pooled ORs were evaluated by comparing the combination of smoking status and NQO1 Pro187Ser or SULT1A1 Arg213His genotypes with the other combination. Furthermore, we used dominant model for NQO1 Pro187Ser and recessive model for SULT1A1 Arg213His to estimate the pooled ORs in this meta-analysis. The Chi²-based Cochran's Q statistic test and $I^{2}$ statistics were employed to test between-study heterogeneity, and heterogeneity was considered significant when $\mathrm{p}<0.05$ for $\mathrm{Q}$ statistic. Fixed-effects model (Mantel-Haenszel method) was used for calculating the pooled ORs when no significant heterogeneity was detected; otherwise, randomeffects model (DerSimonian-Laird method) was applied. Publication bias was assessed by the funnel plot and Egger's test [21]. All p values are two-tailed with a significant level at 0.05 . All statistical analyses were conducted by using STATA 10.0 (Stata Corporation, College Station, USA).

\section{RESULTS}

\section{Characteristics of included studies}

After extensive searching, a total of 7 studies have contained 1341 cases with 1346 controls on NQO1 Pro187Ser and 1921 smoking cases with 1882 controls on SULT$1 A 1 \mathrm{Arg} 213$ His. Smoking is retrieved based on the search criteria for BC susceptibility [12,22-33]. Study characteristics were summarized in the Table 1. For NQO1 Pro187Ser polymorphism and smoking, 4 studies have focused on Caucasian descendants, and 3 studies of Asian descendants. For SULT1A1 Arg213His polymorphism and smoking, 2 studies have focused on Caucasian descendants, and 5 studies of Asian descendants.

\section{Combined effects of gene-smoking for the combined effects of NQO1 Pro187Ser and smoking status}

In the meta-analysis, we did further analysis to explore the potential combined effects between NQO1 Pro187Ser polymorphism and smoking on the risk of bladder cancer which has been shown in the Table 2 and Figure 1. When compared with non-smokers with NQO1 Pro/Pro genotype, non-smokers carried NQO1 Pro/Ser or Ser/Ser genotypes exhibited an increased risk of $\mathrm{BC}$, with OR of 1.76 (95\% CI: 1.34-2.31); and smokers with NQO1 Pro/Pro genotype were observed to confer 2.88 fold increased risk of BC (95\% CI: 2.24-3.70). More importantly, the association was even more prominent for smokers with $N Q O 1$ 
Table 1. Characteristics of studies included in the meta-analysis of NQO1 Pro187Ser or SULT1A1 Arg213His polymorphisms with risk of bladder cancer

\begin{tabular}{|c|c|c|c|c|c|c|c|c|c|}
\hline \multirow{2}{*}{ Study } & \multirow{2}{*}{$\begin{array}{c}\text { Year of } \\
\text { publication }\end{array}$} & \multirow{2}{*}{$\begin{array}{l}\text { Country } \\
\text { (region) }\end{array}$} & \multirow{2}{*}{ Ethnicity } & \multirow{2}{*}{$\begin{array}{l}\text { Control } \\
\text { source }\end{array}$} & \multicolumn{2}{|c|}{$\begin{array}{l}\text { Respondents } \\
{[\mathrm{n}]}\end{array}$} & \multirow{2}{*}{$\begin{array}{l}\text { Genotyping } \\
\text { method }\end{array}$} & \multirow{2}{*}{$\begin{array}{l}\text { Smoking } \\
\text { status }\end{array}$} & \multirow{2}{*}{$\begin{array}{l}\text { HWE in } \\
\text { control } \\
\text { group }\end{array}$} \\
\hline & & & & & $\begin{array}{l}\text { study } \\
\text { group }\end{array}$ & $\begin{array}{l}\text { control } \\
\text { group }\end{array}$ & & & \\
\hline \multicolumn{10}{|l|}{ NQO1 Pro187Ser } \\
\hline Park et al. [22] & 2003 & United States & Caucasian & $\mathrm{HB}$ & 232 & 239 & PCR-RFLP & reported & Y \\
\hline Moore et al. [23] & 2004 & Argentina & Caucasian & PB & 106 & 108 & PCR-RFLP & reported & Y \\
\hline Hung et al. [24] & 2004 & Italy & Caucasian & $\mathrm{HB}$ & 201 & 214 & PCR-RFLP & reported & Y \\
\hline Terry et al. [25] & 2005 & United States & Caucasian & $\mathrm{HB}$ & 239 & 215 & $\begin{array}{l}\text { mass } \\
\text { spectrometry }\end{array}$ & reported & Y \\
\hline Wang et al. [26] & 2008 & China (Taiwan) & Asian & $\mathrm{HB}$ & 300 & 300 & PCR-RFLP & reported & Y \\
\hline Pandith et al. [27] & 2011 & Kashmiri & Asian & $\mathrm{HB}$ & 104 & 120 & PCR-RFLP & reported & Y \\
\hline Huang et al. [28] & 2014 & China & Asian & $\mathrm{HB}$ & 159 & 150 & PCR-RFLP & reported & Y \\
\hline \multicolumn{10}{|l|}{ SULT1A1 Arg213His } \\
\hline Zheng et al. [12] & 2003 & United States & Caucasian & $\mathrm{HB}$ & 384 & 386 & PCR-RFLP & reported & Y \\
\hline Hung et al. [24] & 2004 & Italy & Caucasian & $\mathrm{HB}$ & 201 & 214 & PCR-RFLP & reported & Y \\
\hline Wang et al. [26] & 2008 & China (Taiwan) & Asian & $\mathrm{HB}$ & 300 & 300 & PCR-RFLP & reported & $\mathrm{Y}$ \\
\hline Cui et al. [32] & 2013 & Japan & Asian & $\mathrm{HB}$ & 282 & 257 & PCR-RFLP & reported & Y \\
\hline Tsukino et al. [31] & 2004 & Japan & Asian & $\mathrm{HB}$ & 306 & 306 & PCR-RFLP & reported & Y \\
\hline Ozawa et al. [29] & 2002 & Japan & Asian & $\mathrm{HB}$ & 149 & 189 & PCR-RFLP & reported & $\mathrm{Y}$ \\
\hline Tung et al. [33] & 2014 & China (Taiwan) & Asian & $\mathrm{HB}$ & 299 & 230 & PCR-RFLP & reported & Y \\
\hline
\end{tabular}

NQO1 - quinine oxidoreductase 1; SULT1A1 - sulfotransferase 1A1.

HWE - Hardy-Weinberg equilibrium; HB - hospital-based study; PB - population-based study; PCR-RFLP - polymerase chain reaction-restriction fragment length polymorphism.

Table 2. Summary odds ratios with confidence intervals between smoking-NQO1 Pro187Ser interaction and bladder cancer risk

\begin{tabular}{|c|c|c|c|c|c|c|c|c|}
\hline \multirow{2}{*}{$\begin{array}{l}\text { Smoking-NQO1 } \\
\text { Pro187Ser }\end{array}$} & \multirow{2}{*}{$\begin{array}{l}\text { Studies } \\
{[\mathrm{n}]}\end{array}$} & \multicolumn{2}{|c|}{$\begin{array}{l}\text { Respondents } \\
{[\mathrm{n}]}\end{array}$} & \multicolumn{2}{|c|}{ Heterogeneity } & \multirow{2}{*}{$\begin{array}{l}\text { Model for } \\
\text { meta-analysis }\end{array}$} & \multirow{2}{*}{ OR $(95 \% \mathrm{CI})$} & \multirow{2}{*}{$\mathrm{p}_{\text {Egger's test }}{ }^{\mathrm{b}}$} \\
\hline & & $\begin{array}{l}\text { study } \\
\text { group }\end{array}$ & $\begin{array}{c}\text { control } \\
\text { group }\end{array}$ & $\begin{array}{c}\mathrm{I}^{2} \\
{[\%]}\end{array}$ & $\mathrm{p}_{\text {heterogneity }}$ & & & \\
\hline$-/(\mathrm{CT}+\mathrm{TT})$ vs. $-/ \mathrm{CC}$ & 7 & 398 & 634 & 0.0 & 0.590 & $\mathrm{~F}$ & $1.76(1.34-2.31)$ & 0.049 \\
\hline$+/ \mathrm{CC}$ vs. $-/ \mathrm{CC}$ & 7 & 623 & 733 & 41.1 & 0.117 & $\mathrm{~F}$ & $2.88(2.24-3.70)$ & 0.387 \\
\hline$+/(\mathrm{CT}+\mathrm{TT})$ vs. $-/ \mathrm{CC}$ & 7 & 594 & 620 & 6.7 & 0.376 & $\mathrm{~F}$ & $3.71(2.87-4.79)$ & 0.092 \\
\hline$+/(\mathrm{CT}+\mathrm{TT})$ vs. $-/(\mathrm{CT}+\mathrm{TT})$ & 7 & 714 & 612 & 32.4 & 0.181 & $\mathrm{~F}$ & $1.99(1.48-2.69)$ & 0.683 \\
\hline$+/(\mathrm{CT}+\mathrm{TT})$ vs. $+/ \mathrm{CC}$ & 7 & 939 & 711 & 66.4 & 0.007 & $\mathrm{R}$ & $1.34(0.93-1.95)$ & 0.423 \\
\hline
\end{tabular}

NQO1 - quinine oxidoreductase 1.

$\mathrm{OR}$ - odds ratio; $\mathrm{CI}$ - confidence interval.

$\mathrm{F}$ - fixed-effects model; $\mathrm{R}$ - random-effects model.

${ }^{a} \mathrm{p}$ value of Q-test for heterogeneity test.

${ }^{\mathrm{b}} \mathrm{p}$ value for Egger's test. 


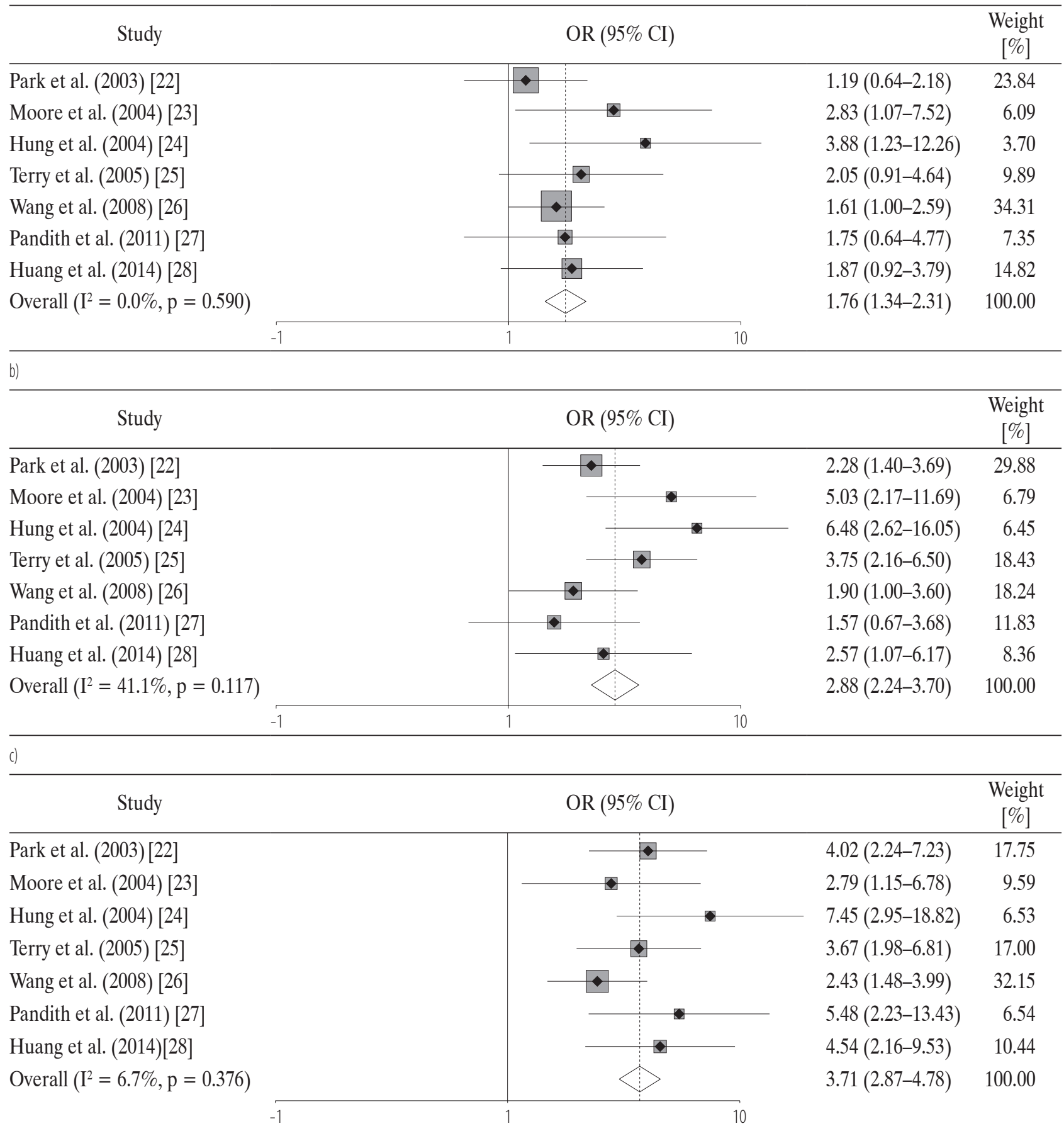

NQO1 - quinine oxidoreductase 1.

$\mathrm{OR}$ - odds ratio; $\mathrm{CI}$ - confidence interval.

Fig. 1. Forest plot of the combined effects of NQO1 Pro187Ser polymorphism and smoking on the risk of bladder cancer:

a) non-smokers carried NQO1 Pro/Ser or Ser/Ser genotypes vs. non-smokers with NQO1 Pro/Pro genotype,

b) smokers carried NQO1 Pro/Pro genotype vs. non-smokers with NQO1 Pro/Pro genotype,

c) smokers carried NQO1 Pro/Ser or Ser/Ser genotypes vs. non-smokers with NQO1 Pro/Pro genotype 
Pro/Ser or Ser/Ser genotypes compared with non-smokers with NQO1 Pro/Pro genotype, with OR equal to 3.71 (95\% CI: 2.87-4.78).

In addition, we also compared smokers with NQO1 Pro/ Ser or Ser/Ser genotypes with non-smoker carrying NQO1 Pro/Ser or Ser/Ser genotypes, the associated OR equaled 1.99 (95\% CI: 1.48-2.69). Among smokers, NQO1 Pro/ Ser or Ser/Ser genotypes showed no significant association with the risk of BC when compared with NQO1 Pro/Pro genotype (OR $=1.34,95 \%$ CI: 0.93-1.95).

\section{For the combined effects}

\section{of SULT1A1 Arg213His and smoking status}

The Table 3 and Figure 2 show the potentially combined effects between SULT1A1 Arg213His polymorphism and smoking on the risk of bladder cancer. When compared with non-smokers with SULT1A1 Arg/His or His/His genotypes, non-smokers carrying SULT1A1 Arg/Arg show an increased risk of BC, with OR of 1.52 (95\% CI: 1.181.96); and smokers carrying SULT1A1 Arg/His or His/His genotypes with the OR of 2.00 (95\% CI: 1.54-2.60) show the increased risk of $\mathrm{BC}$. Interestingly, smokers carrying
SULT1A1 Arg/Arg genotypes have been observed to confer 2.38 fold increased risk of BC (95\% CI: 1.44-3.93). Similarly, we have also compared smokers with SULT$1 A 1 \mathrm{Arg} / \mathrm{Arg}$ genotypes with non-smokers carrying SULT$1 A 1 \mathrm{Arg} / \mathrm{Arg}$, the associated OR equals 1.49 (95\% CI: 1.27-1.75). Among smokers, SULTIA1 Arg/Arg genotypes show significant association with the risk of $\mathrm{BC}$ when compared with SULT1A1 Arg/His or His/His genotypes $(\mathrm{OR}=1.21,95 \%$ CI: $1.01-1.46)$.

\section{Publication bias}

Begg's funnel plot (Figure 3) and Egger's test were performed to assess the publication bias in related literature (Table 2 and Table 3). For NQO1 Pro187Ser and smoking status, the shape of funnel plots seemed asymmetrical for non-smokers carrying NQO1 Pro/Ser or Ser/Ser genotypes vs. non-smokers, with p, that was used in Egger's test, equaling 0.049. Besides, the shape of the funnel plots for smokers carrying NQO1 Pro/Pro genotype vs. nonsmokers with NQO1 Pro/Pro genotype did not show either any evidence of the funnel plot asymmetry or publication bias detected by using Egger's test, with p values equaling

Table 3. Summary odds ratios with confidence intervals between smoking-SULT1A1 Arg213His interaction and bladder cancer risk

\begin{tabular}{|c|c|c|c|c|c|c|c|c|}
\hline \multirow{2}{*}{$\begin{array}{l}\text { Smoking-SULT1A1 } \\
\text { Arg213His }\end{array}$} & \multirow{2}{*}{$\begin{array}{l}\text { Studies } \\
{[\mathrm{n}]}\end{array}$} & \multicolumn{2}{|c|}{$\begin{array}{l}\text { Respondents } \\
{[\mathrm{n}]}\end{array}$} & \multicolumn{2}{|c|}{ Heterogeneity } & \multirow{2}{*}{$\begin{array}{l}\text { Model for } \\
\text { meta-analysis }\end{array}$} & \multirow{2}{*}{ OR (95\% CI) } & \multirow{2}{*}{$\mathrm{p}_{\text {Egger's test }}{ }^{\mathrm{b}}$} \\
\hline & & $\begin{array}{l}\text { study } \\
\text { group }\end{array}$ & $\begin{array}{l}\text { control } \\
\text { group }\end{array}$ & $\begin{array}{c}\mathrm{I}^{2} \\
{[\%]}\end{array}$ & $\mathrm{p}_{\text {heterogneity }}{ }^{\mathrm{a}}$ & & & \\
\hline $\begin{array}{l}-/(\mathrm{Arg} / \mathrm{Arg}) \\
\text { vs. }-/(\mathrm{Arg} / \mathrm{His}+\mathrm{His} / \mathrm{His})\end{array}$ & 7 & 652 & 819 & 22.8 & 0.256 & $\mathrm{~F}$ & $1.52(1.18-1.96)$ & 0.777 \\
\hline $\begin{array}{l}+/(\operatorname{Arg} / \operatorname{Arg}+\mathrm{His} / \mathrm{His}) \\
\quad \text { vs. }-/(\mathrm{Arg} / \mathrm{His}+\mathrm{His} / \mathrm{His})\end{array}$ & 7 & 502 & 596 & 49.2 & 0.066 & $\mathrm{~F}$ & $2.00(1.54-2.60)$ & 0.834 \\
\hline $\begin{array}{l}+/(\operatorname{Arg} / \operatorname{Arg}) \\
\text { vs. }-/(\operatorname{Arg} / \mathrm{His}+\mathrm{His} / \mathrm{His})\end{array}$ & 7 & 1039 & 973 & 74.7 & 0.001 & $\mathrm{R}$ & $2.38(1.44-3.93)$ & 0.530 \\
\hline $\begin{array}{l}+/(\operatorname{Arg} / \operatorname{Arg}) \\
\text { vs. }-/(\operatorname{Arg} / \operatorname{Arg})\end{array}$ & 7 & 1419 & 1286 & 46.5 & 0.082 & $\mathrm{~F}$ & $1.49(1.27-1.75)$ & 0.074 \\
\hline $\begin{array}{l}+/(\operatorname{Arg} / \mathrm{Arg}) \\
\quad \text { vs. }+/(\mathrm{Arg} / \mathrm{His}+\mathrm{His} / \mathrm{His})\end{array}$ & 7 & 1269 & 1063 & 0.0 & 0.683 & $\mathrm{~F}$ & $1.21(1.01-1.46)$ & 0.256 \\
\hline
\end{tabular}

Abbreviations as in Tables 1 and 2. 


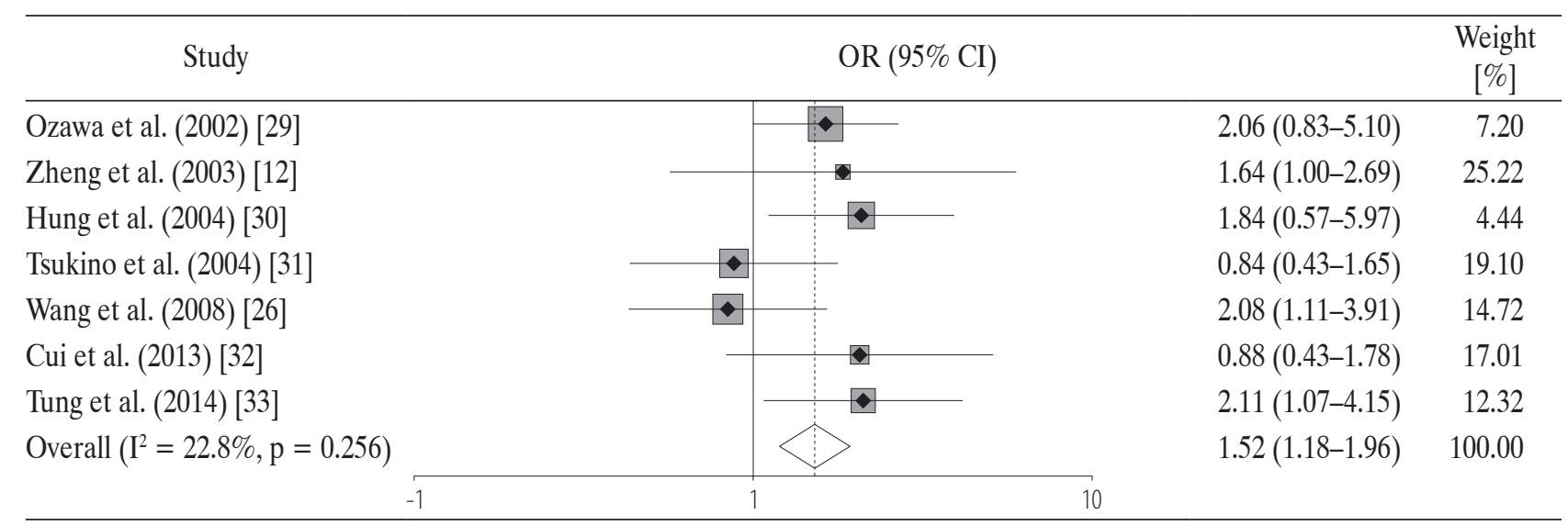

b)

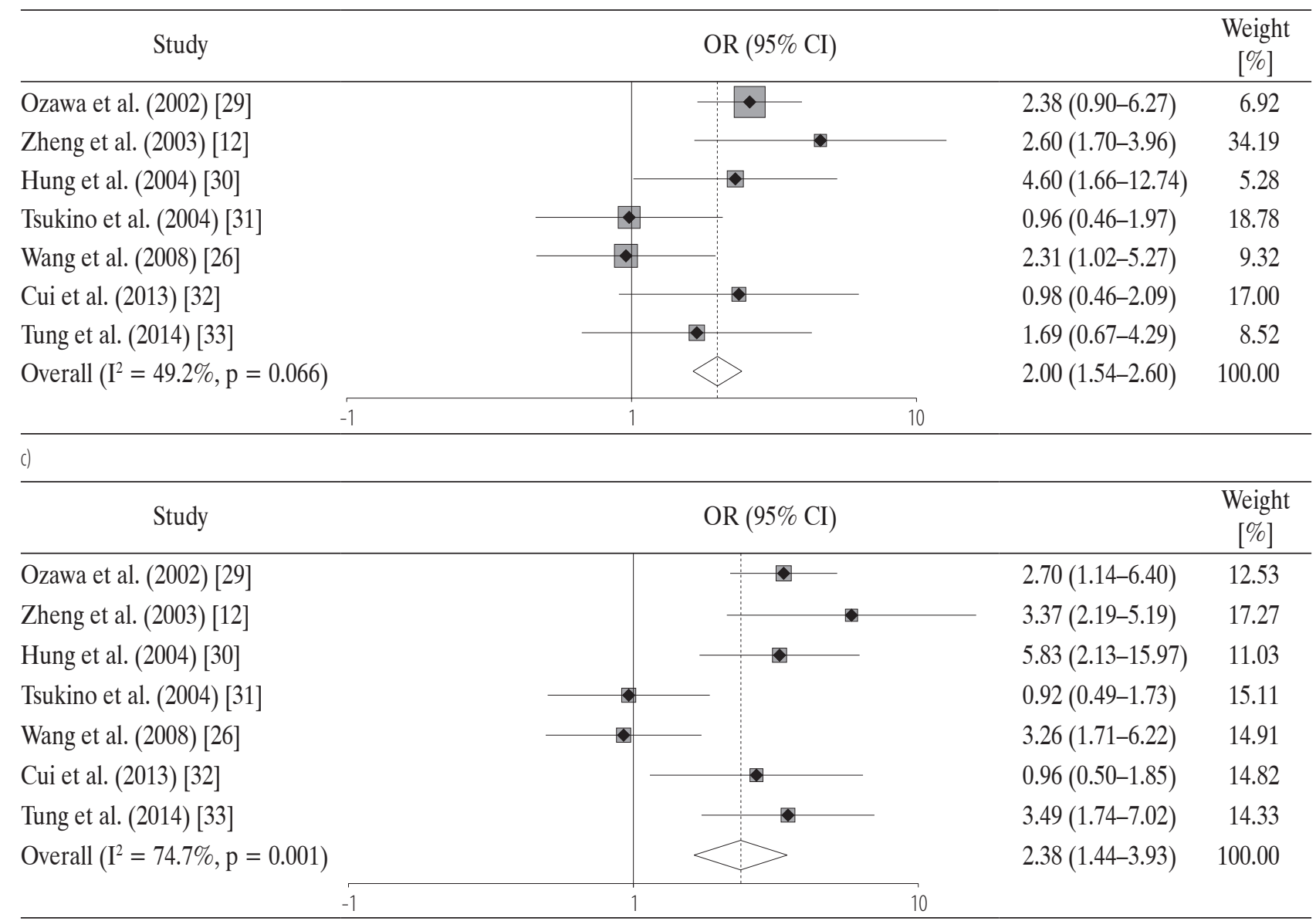

SULT1A1 - sulfotransferase 1A1.

OR - odds ratio; $\mathrm{CI}$ - confidence interval.

Fig. 2. Forest plot of the combined effects of SULT1A1 Arg213His polymorphism and smoking on the risk of bladder cancer:

a) non-smokers carried SULT1A1 Arg/Arg genotypes vs. non-smokers with SULT1A1 Arg/His or His/His genotype,

b) smokers with SULT1A1 Arg/His or His/His genotype vs. non-smokers with SULT1A1 Arg/His or His/His genotype,

c) smokers with SULT1A1 Arg/Arg vs. non-smokers with SULT1A1 Arg/His or His/His genotypes 

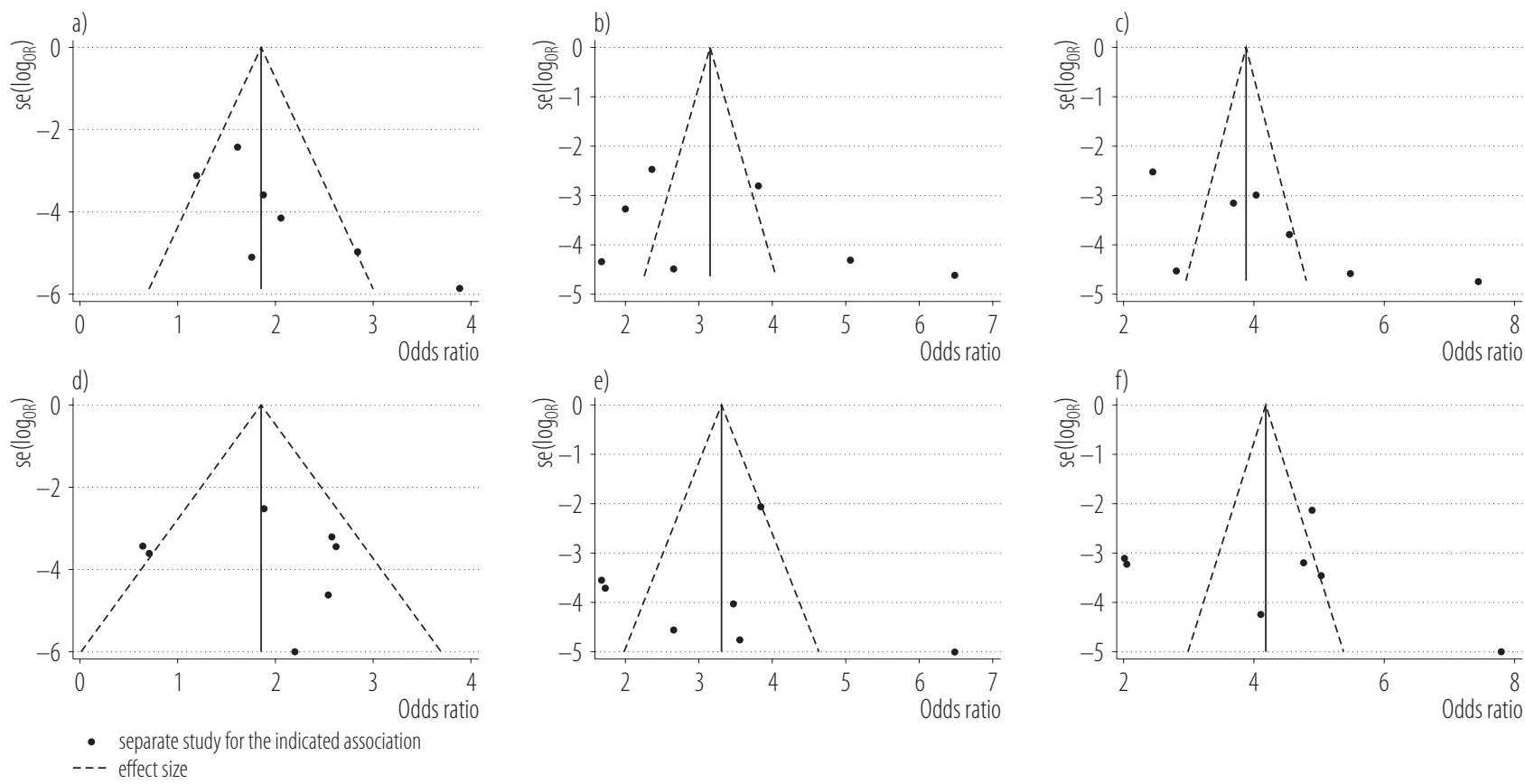

$\log _{\mathrm{OR}}$ - natural logarithm of odds ratio (OR).

Fig. 3. Begg's funnel plot for publication bias test - smoking-NQO1 Pro187Ser interaction: a) -/(CT+TT) vs. -/CC, b) +/CC vs. -/CC; c) +/(CT+TT) vs. -/CC; smoking-SULT1A1 Arg213His interaction: d) -/(Arg/Arg) vs. -/(Arg/His+His/His); e) +/(Arg/Arg + His/

His) vs. -/(Arg/His+His/His); f) +/(Arg/Arg) vs. -/(Arg/His+His/His)

0.387. Similarly, the shape of funnel plots seemed symmetrical for smokers carrying NQO1 Pro/Ser or Ser/Ser genotypes vs. non-smokers with NQO1 Pro/Pro genotype, with $\mathrm{p}$, that was used in Egger's test, equaling 0.092.

For SULT1A1 Arg213His and smoking status, all the shapes of funnel plots seemed symmetrical, with $\mathrm{p}$, that was used in Egger's test, equaling 0.777 for non-smokers carrying SULT1A1 Arg/Arg genotypes vs. non-smokers with, 0.834 for smokers with SULT1A1 Arg/His or His/ His genotype vs. non-smokers with, and 0.530 for smokers with SULT1A1 Arg/Arg vs. non-smokers with SULT$1 A 1 \mathrm{Arg} / \mathrm{His}$ or His/His genotype.

\section{DISCUSSION}

We conducted 2 meta-analyses including 1341 cases and 1346 controls concerning NQO1 Pro187Ser and smoking, and 1921 cases and 1882 controls on SULT1A1 Arg213His and smoking. The combined effects of the 2 missense polymorphisms and smoking on bladder cancer risk were firstly explored by meta-analyses. The result demonstrated that smokers with NQO1 Pro/Ser or Ser/Ser genotypes had a prominent association with the risk of $\mathrm{BC}$ as compared with non-smokers with NQO1 Pro/Pro genotype, with OR equaling 3.71 (95\% CI: 2.87-4.78). Besides, smokers carrying SULT1A1 Arg/Arg genotypes were observed to confer 2.38 fold increased risk of BC (95\% CI: 1.44-3.93) when compared with non-smokers with SULT1A1 Arg/Arg or His/His genotypes.

The NQO1 enzyme has been phase II enzyme and involves in the detoxification of chemical carcinogens generated from cigarette smoking. So far, several previous studies have investigated the genetic effect of $N Q O 1$ Pro187Ser modified by smoking on BC risk. However, the reported associations of NQO1 Pro187Ser and smoking with $\mathrm{BC}$ risk have been inconsistent. As far as the limited power of the individual studies is concerned, we 
have analyzed the pooled effect of Pro187Ser and smoking on the risk of $\mathrm{BC}$ by integrating individual studies. In the meta-analysis, non-smokers carrying NQO1 Pro/ Ser or Ser/Ser genotypes have exhibited an increased risk of BC, with OR at 1.76 (95\% CI: 1.34-2.31) as compared with non-smokers with NQO1 Pro/Pro genotype whereas smokers with NQO1 Pro/Ser or Ser/Ser genotypes have shown no significant association with the risk of $\mathrm{BC}$ as compared to smokers with NQO1 Pro/Pro genotype (OR $=1.34,95 \%$ CI: 0.93-1.95).

However, a similar trend has been observed in the case of non-smokers and smokers. The joint effects between NQO1 Pro187Ser and smoking have been observed. Smokers with NQO1 Pro/Ser or Ser/Ser genotypes have shown more prominent association with the risk of $\mathrm{BC}$ as compared with non-smokers with NQO1 Pro/Pro genotype, with OR equaling 3.71 (95\% CI: 2.87-4.78). The joint effect has suggested that NQO1 Pro/Ser or Ser/Ser genotypes confer some benefit for smokers as opposed to smokers with the Pro/ Pro genotype. Additionally, publication biases have been comprehensively examined by using Begg's funnel plot and Egger's tests. Border line publication bias has only been detected for non-smokers carrying NQO1 Pro/Ser or Ser/Ser genotypes vs. non-smokers $(\mathrm{p}=0.049)$ and no publication bias has been detected for the other comparison. In view of this, we are convinced that the results of our meta-analysis, in essence, are sound and reliable.

Sulfotransferase $1 A 1$ which is involved in detoxification pathways is responsible for metabolizing a wide range of endogenous and exogenous carcinogens. The genotypes containing SULT1A1 His213 allele have been observed less efficiently at DNA adduct formation than SULT1A1 Arg/Arg genotype [34]. Moreover, results of the previous meta-analysis have proven that SULT1A1 Arg/Arg genotype is significantly associated with increased BC risk [17,18]. The combined effects of SULT1A1 Arg213His and smoking on $\mathrm{BC}$ risk have been investigated by individual studies, but the result has not been inconsistent.

\section{CONCLUSIONS}

Therefore, we conducted a meta-analysis to estimate the pooled effect of SULT1A1 Arg213His and smoking on the risk of $\mathrm{BC}$ by integrating individual studies. The results show that non-smokers carrying SULT1A1 Arg/Arg show an increased risk of BC, with OR at 1.52 (95\% CI: 1.18-1.96) when compared with non-smokers with SULT1A1 Arg/His or His/His genotypes. Among smokers, SULT1A1 Arg/Arg genotypes also show significant association with the risk of $\mathrm{BC}$ as compared with SULT1A1 Arg/His or His/His genotypes $(\mathrm{OR}=1.21,95 \%$ CI: 1.01-1.46). We can see from the result that SULT1A1 Arg/Arg genotype is consistently associated with increased $\mathrm{BC}$ risk when stratified by smoking status.

Meanwhile, smokers carrying SULT1A1 Arg/Arg genotypes have been observed to confer 2.38 fold increased risk of BC as compared with non-smokers with SULT1A1 $\mathrm{Arg} / \mathrm{His}$ or His/His genotypes. The joint effect of SULT$1 A 1 \mathrm{Arg} 213 \mathrm{His}$ and smoking has been observed from the result. We have noted that the effect of SULT1A1 Arg213His polymorphism on BC risk is likely to be enhanced in relation to smoking. Fortunately, biological plausibility exists for an elucidation of the combined effect between SULT1A1 Arg213His and smoking [13,33]. Similarly, publication biases have been comprehensively examined by using Begg's funnel plot and Egger's tests. No publication bias has been detected for all the comparison about the combined effect of smoking and SULT1A1 Arg213His. Therefore, the results of our meta-analysis are sound and reliable.

To the best of our knowledge, the 2 meta-analyses have firstly explored the combined effects of NQO1 Pro187Ser or SULT1A1 Arg213His polymorphisms and smoking on $\mathrm{BC}$ risk, which seem biologically plausible. The strengths of our meta-analysis could be summarized as follows: firstly, the publications have been systematically browsed through by various searching approaches and satisfactory studies, which have met our inclusion criterion, have been finally selected. Secondly, all the comparisons 
about the combined effect of NQO1 Pro187Ser or SULT$1 A 1$ Arg213His polymorphisms and smoking have been performed to estimate the pooled OR.

Besides, the effect of NQO1 Pro187Ser or SULT1A1 Arg213 His polymorphisms stratified by smoking has also been examined by combining all the included studies. Our results further provide some support that NQO1 Pro187Ser or SULT1A1 Arg213His polymorphism combination with smoking significantly increase the risk of $\mathrm{BC}$. The biological function analysis should be performed to validate the combined effect between NQO1 Pro187Ser or SULT$1 A 1 \mathrm{Arg} 213$ His polymorphism and smoking in modulation of $\mathrm{BC}$ risk. Furthermore, well-designed studies with a sufficient sample size are warranted to further confirm the associated OR modified by smoking.

\section{REFERENCES}

1. Jemal A, Bray F, Center MM, Ferlay J, Ward E, Forman D. Global cancer statistics. CA Cancer J Clin. 2011;61:69-90, https://doi.org/10.3322/caac.20107.

2. Kaufman DS, Shipley WU, Feldman AS. Bladder cancer. Lancet. 2009;374:239-49, https://doi.org/10.1016/S0140-6736 (09)60491-8.

3. Kalble T. [Etiopathology, risk factors, environmental influences and epidemiology of bladder cancer]. Urologe A. 2001;40: 447-50, https://doi.org/10.1007/s001200170003. German.

4. Freedman ND, Silverman DT, Hollenbeck AR, Schatzkin A, Abnet CC. Association between smoking and risk of bladder cancer among men and women. JAMA. 2011;306:737-45, https://doi.org/10.1001/jama.2011.1142.

5. Brennan P, Bogillot O, Cordier S, Greiser E, Schill W, Vineis $\mathrm{P}$, et al. Cigarette smoking and bladder cancer in men: A pooled analysis of 11 case-control studies. Int J Cancer. 2000;86:289-94, https://doi.org/10.1002/(SICI)1097-0215 (20000415)86:2<289::AID-IJC21>3.0.CO;2-M.

6. Hoffmann D, Hoffmann I. The changing cigarette, 19501995. J Toxicol Environ Health. 1997;50:307-64, https://doi. org/10.1080/009841097160393.
7. Vineis P, Talaska G, Malaveille C, Bartsch H, Martone T, Sithisarankul P, et al. DNA adducts in urothelial cells: Relationship with biomarkers of exposure to arylamines and polycyclic aromatic hydrocarbons from tobacco smoke. Int J Cancer. 1996;65:314-6, https://doi.org/10.1002/(SICI)10970215(19960126)65:3<314::AID-IJC6>3.0.CO;2-2.

8. Ross D, Kepa JK, Winski SL, Beall HD, Anwar A, Siegel D. NAD(P)H:quinone oxidoreductase 1 (NQO1): Chemoprotection, bioactivation, gene regulation and genetic polymorphisms. Chem Biol Interact. 2000;129:77-97, https://doi. org/10.1016/S0009-2797(00)00199-X

9. Nebert DW, Roe AL, Vandale SE, Bingham E, Oakley GG. NAD(P)H:quinone oxidoreductase (NQO1) polymorphism, exposure to benzene, and predisposition to disease: A HuGE review. Genet Med. 2002;4:62-70, https://doi. org/10.1097/00125817-200203000-00003.

10. Coughtrie MW, Johnston LE. Interactions between dietary chemicals and human sulfotransferases-molecular mechanisms and clinical significance. Drug Metab Dispos. 2001;29:522-8.

11. Traver RD, Horikoshi T, Danenberg KD, Stadlbauer TH, Danenberg PV, Ross D, et al. NAD(P)H:quinone oxidoreductase gene expression in human colon carcinoma cells: Characterization of a mutation which modulates DT-diaphorase activity and mitomycin sensitivity. Cancer Res. 1992;52:797-802.

12. Zheng L, Wang Y, Schabath MB, Grossman HB, Wu X. Sulfotransferase 1A1 (SULT1A1) polymorphism and bladder cancer risk: A case-control study. Cancer Lett. 2003;202: 61-9, https://doi.org/10.1016/j.canlet.2003.08.007.

13. Pereira WO, Paiva AS, Queiroz JW, Toma L, Dietrich CP, Nader HB, et al. Genetic polymorphism in the sulfotransferase SULT1A1 gene in cancer. Cancer Genet Cytogenet. 2005;160:55-60, https://doi.org/10.1016/j.cancergencyto.2004. 12.005 .

14. Gong M, Yi Q, Wang W. Association between NQO1 C609T polymorphism and bladder cancer susceptibility: A systemic review and meta-analysis. Tumour Biol. 2013;34:2551-6, https://doi.org/10.1007/s13277-013-0799-7. 
15. Lajin B, Alachkar A. The NQO1 polymorphism C609T (Pro187Ser) and cancer susceptibility: A comprehensive meta-analysis. Br J Cancer. 2013;109:1325-37, https://doi. org/10.1038/bjc.2013.357.

16. Mandal RK, Dubey S, Panda AK, Mittal RD. Genetic variants of NQO1 gene increase bladder cancer risk in Indian population and meta-analysis. Tumour Biol. 2014;35:641523, https://doi.org/10.1007/s13277-014-1869-1.

17. Li W, Gu M. SULT1A1 Arg213His polymorphism is associated with bladder cancer risk: A meta-analysis. Med Sci Monit. 2014;20:1590-5, https://doi.org/10.12659/MSM.890822.

18. Su CM, Chen MC, Lin IC, Chen HA, Huang MT, Wu CH, et al. Association between the SULT1A1 Arg213His polymorphism and the risk of bladder cancer: A meta-analysis. Tumour Biol. 2014;35:7147-53, https://doi.org/10.1007/s13 277-014-1954-5.

19. Yang S, Jin T, Su HX, Zhu JH, Wang DW, Zhu SJ, et al. The association between NQO1 Pro187Ser polymorphism and bladder cancer susceptibility: A meta-analysis of 15 studies. PLoS One. 2015;10:e116500, https://doi.org/10.1371/journal. pone. 0116500 .

20. Zhang Y, Yang D, Zhu JH, Chen MB, Shen WX, He J. The association between NQO1 Pro187Ser polymorphism and urinary system cancer susceptibility: A meta-analysis of 22 studies. Cancer Invest. 2015;33:39-40, https://doi.org/ 10.3109/07357907.2014.998836.

21. Egger M, Davey SG, Schneider M, Minder C. Bias in meta-analysis detected by a simple, graphical test. BMJ. 1997;315:629-34, https://doi.org/10.1136/bmj.315.7109.629.

22. Park SJ, Zhao H, Spitz MR, Grossman HB, Wu X. An association between $N Q O 1$ genetic polymorphism and risk of bladder cancer. Mutat Res. 2003;536:131-7, https://doi. org/10.1016/S1383-5718(03)00041-X.

23. Moore LE, Wiencke JK, Bates MN, Zheng S, Rey OA, Smith AH. Investigation of genetic polymorphisms and smoking in a bladder cancer case-control study in Argentina. Cancer Lett. 2004;211:199-207, https://doi.org/10.1016/ j.canlet.2004.04.011.
24. Hung RJ, Boffetta P, Brennan P, Malaveille C, Gelatti U, Placidi D, et al. Genetic polymorphisms of MPO, COMT, MnSOD, NQO1, interactions with environmental exposures and bladder cancer risk. Carcinogenesis. 2004;25:973-8, https://doi.org/10.1093/carcin/bgh080.

25. Terry PD, Umbach DM, Taylor JA. No association between SOD2 or NQO1 genotypes and risk of bladder cancer. Cancer Epidemiol Biomarkers Prev. 2005;14:753-4, https://doi. org/10.1158/1055-9965.EPI-04-0574.

26. Wang YH, Lee YH, Tseng PT, Shen CH, Chiou HY. Human $\mathrm{NAD}(\mathrm{P}) \mathrm{H}$ :quinone oxidoreductase 1 (NQO1) and sulfotransferase 1A1 (SULT1A1) polymorphisms and urothelial cancer risk in Taiwan. J Cancer Res Clin Oncol. 2008;134:203-9, https://doi.org/10.1007/s00432-007-0271-4.

27. Pandith AA, Khan NP, Shah ZA, Shah AM, Wani SM, Siddiqi MA. Association of bladder cancer risk with an $\mathrm{NAD}(\mathrm{P}) \mathrm{H}$ :quinone oxidoreductase polymorphism in an ethnic Kashmiri population. Biochem Genet. 2011;49:417-26, https://doi.org/10.1007/s10528-011-9418-8.

28. Huang ZM, Chen HA, Chiang YT, Shen CH, Tung MC, Juang GD. Association of polymorphisms in iNOS and NQO1 with bladder cancer risk in cigarette smokers. J Chin Med Assoc. 2014;77:83-8, https://doi.org/10.1016/ j.jcma.2013.10.005.

29. Ozawa S, Katoh T, Inatomi H, Imai H, Kuroda Y, Ichiba M, et al. Association of genotypes of carcinogen-activating enzymes, phenol sulfotransferase SULT1A1 (ST1A3) and arylamine N-acetyltransferase NAT2, with urothelial cancer in a Japanese population. Int J Cancer. 2002;102: 418-21, https://doi.org/10.1002/ijc.10728.

30. Hung RJ, Boffetta P, Brennan P, Malaveille C, Hautefeuille A, Donato F, et al. GST, NAT, SULT1A1, CYP1B1 genetic polymorphisms, interactions with environmental exposures and bladder cancer risk in a high-risk population. Int J Cancer. 2004;110:598-604, https://doi.org/10.1002/ijc.20157.

31. Tsukino H, Kuroda Y, Nakao H, Imai H, Inatomi H, Osada Y, et al. Cytochrome P450 (CYP) 1A2, sulfotransferase (SULT) 1A1, and N-acetyltransferase (NAT) 2 polymorphisms and 
susceptibility to urothelial cancer. J Cancer Res Clin Oncol. 2004;130:99-106, https://doi.org/10.1007/s00432-003-0512-0.

32. Cui X, Lu X, Hiura M, Omori H, Miyazaki W, Katoh T. Association of genotypes of carcinogen-metabolizing enzymes and smoking status with bladder cancer in a Japanese population. Environ Health Prev Med. 2013;18:136-42, https:// doi.org/10.1007/s12199-012-0302-x.

33. Tung MC, Wang YH, Yeh SD, Wu CC, Chen KC, Huang ZM, et al. Combined effects of GSTO1 and SULT1A1 polymor- phisms and cigarette smoking on urothelial carcinoma risk in a Taiwanese population. J Formos Med Assoc. 2014;113: 640-7, https://doi.org/10.1016/j.jfma.2012.08.015.

34. Nowell S, Ambrosone CB, Ozawa S, MacLeod SL, Mrackova G, Williams S, et al. Relationship of phenol sulfotransferase activity (SULT1A1) genotype to sulfotransferase phenotype in platelet cytosol. Pharmacogenetics. 2000;10:789-97, https://doi.org/10.1097/00008571-200012000-00004.

This work is available in Open Access model and licensed under a Creative Commons Attribution-NonCommercial 3.0 Poland License - http://creativecommons.org/ licenses/by-nc/3.0/pl/deed.en. 